\title{
Zwitterionic Hydrogels Incorporated Graphene Oxide Nanosheets with Improved Strength and Lubricity
}

Zhongnan Wang, ${ }^{a}$ Jinjin Li, ${ }^{\text {a* }}$ Liang Jiang, ${ }^{\mathrm{b}}$ Shun Xiao, ${ }^{\mathrm{c}}$ Yuhong Liu, ${ }^{\text {a* }}$ and Jianbin Luo ${ }^{\mathrm{a}}$

${ }^{a}$ State Key Laboratory of Tribology, Tsinghua University, Beijing 100084, China.

${ }^{b}$ Tribology Research Institute, State Key Laboratory of Traction Power, Southwest Jiaotong University, Chengdu 610031, China.

${ }^{c}$ School of Biomedical Engineering, Shanghai Jiao Tong University, Shanghai 200030, China.

Corresponding authors:

*To whom all correspondence should be addressed.

Jinjin Li

E-mail: lijinjin@mail.tsinghua.edu.cn

Yuhong Liu

E-mail: liuyuhong@tsinghua.edu.cn 
1. Rheological properties of hydrogels

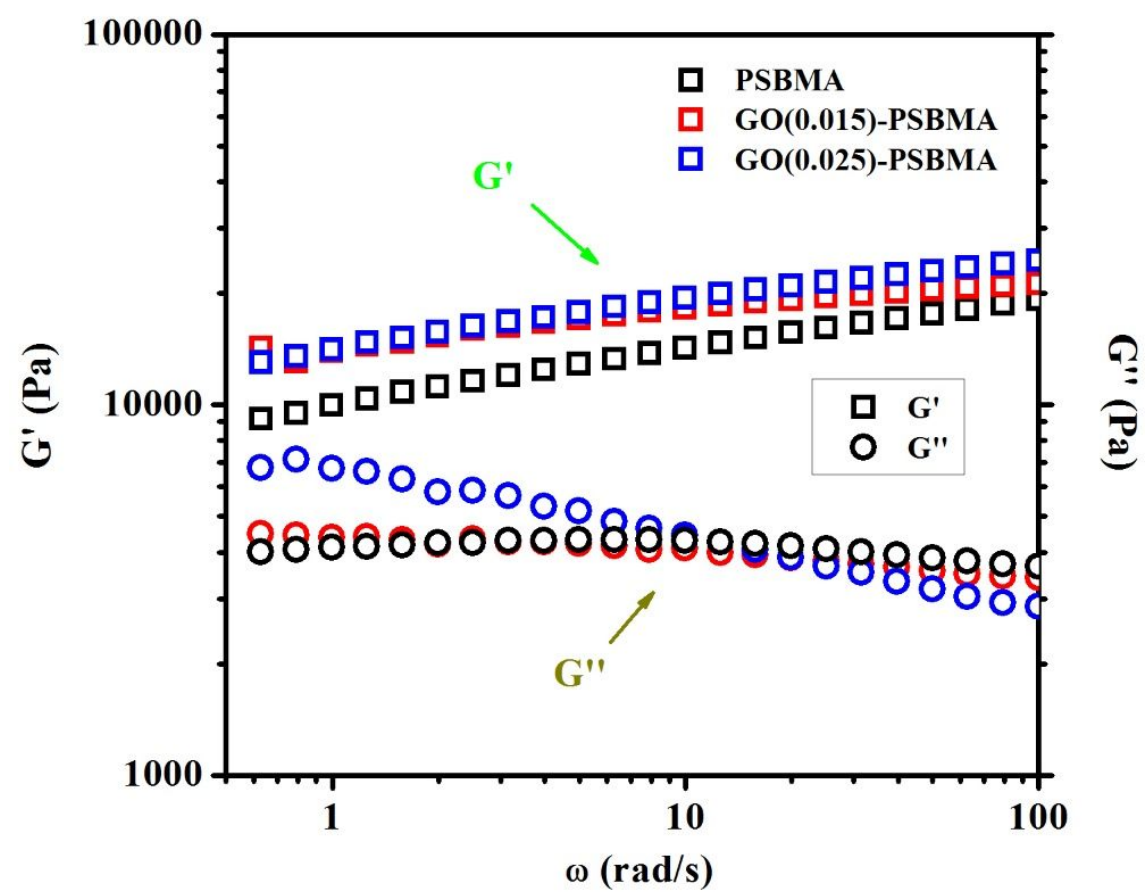

Figure S1 Dynamic moduli of PSBMA hydrogel and GO-PSBMA nanocomposite hydrogel with different GO concentrations (0.015 wt.\% and 0.025 wt.\%) as a function of angular frequency at the temperature of $25^{\circ} \mathrm{C}$. 
2. Gelation time of hydrogels

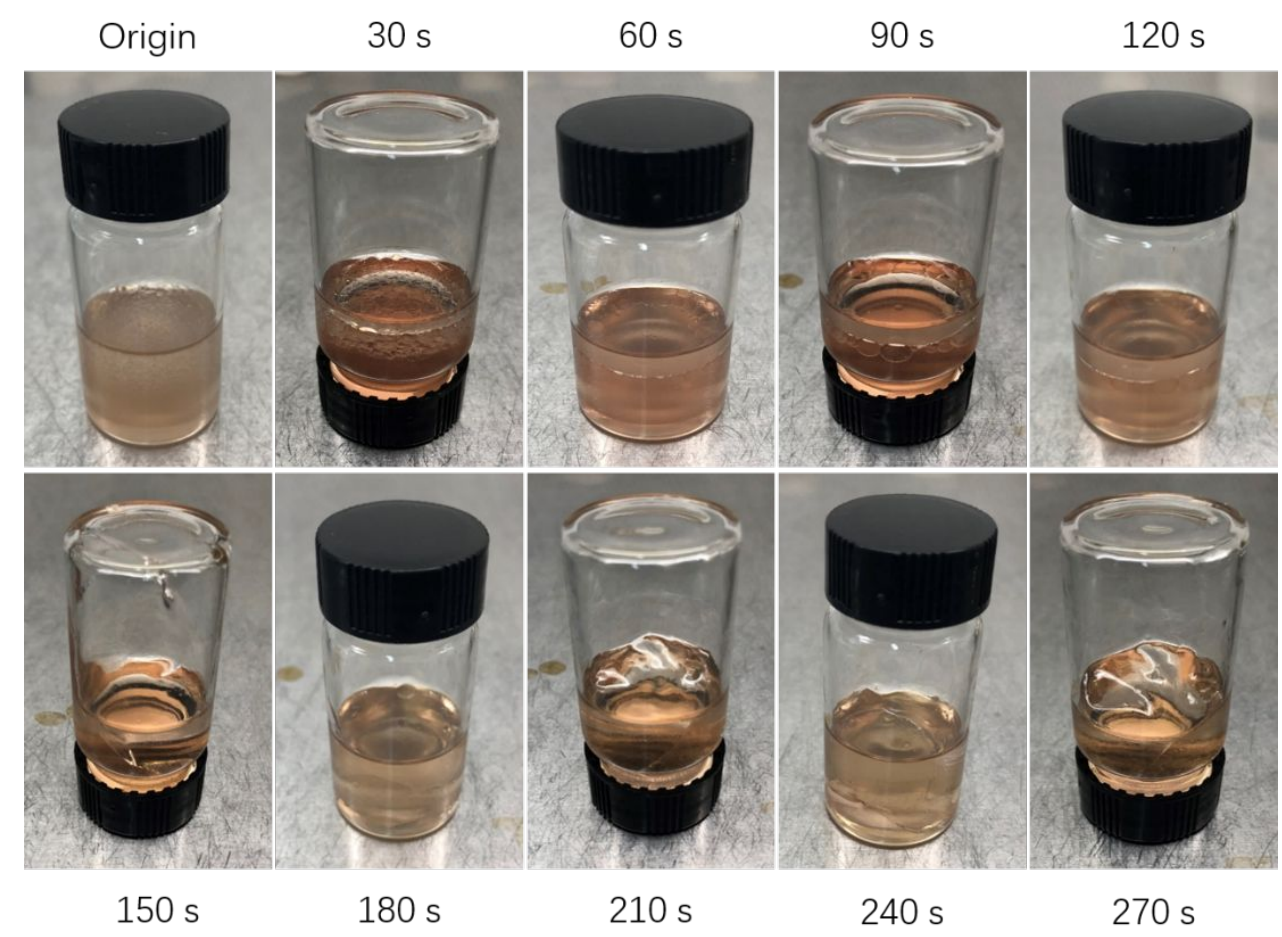

(a)

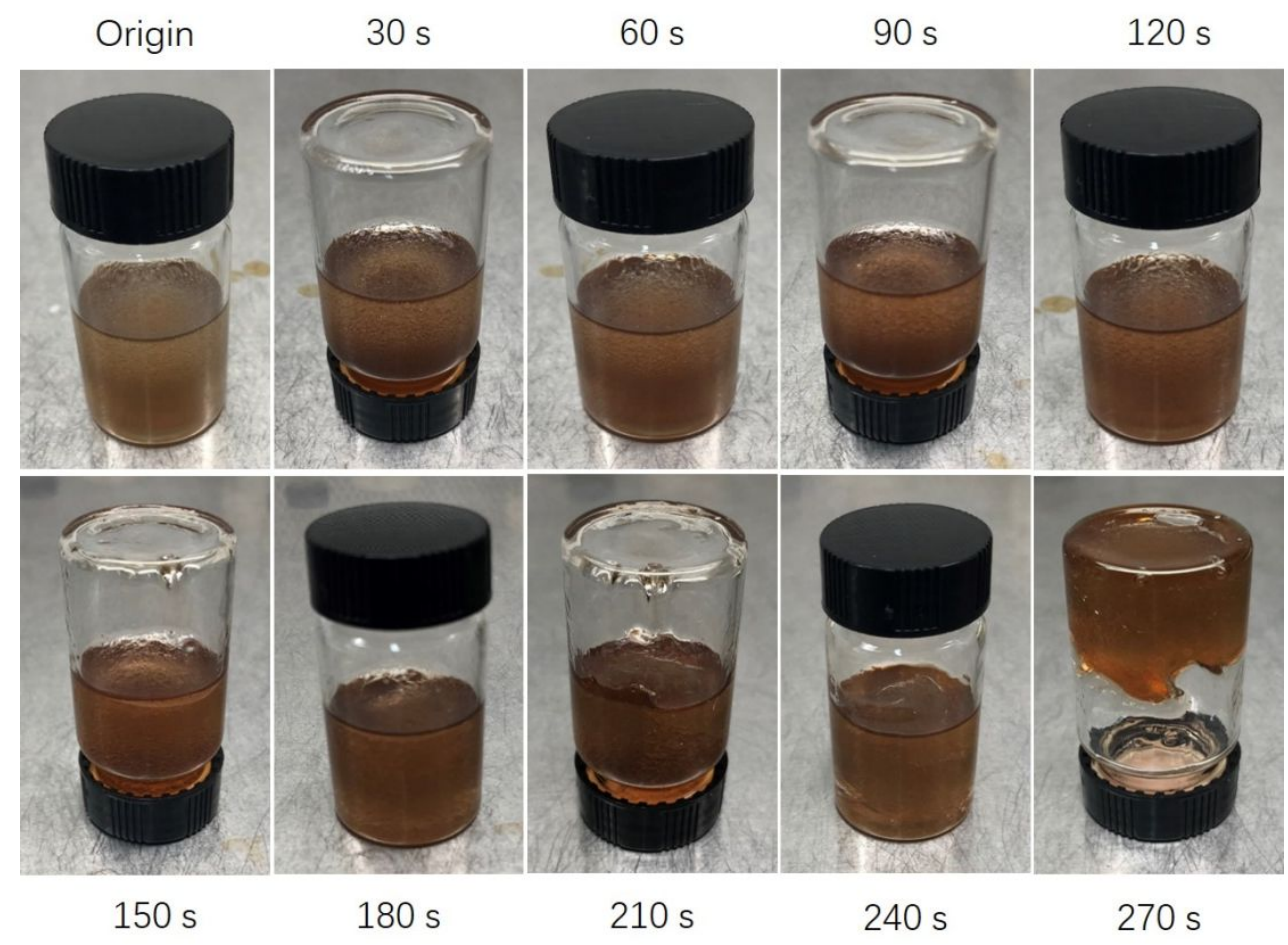

(b)

Figure S2 (a) gelation process of PSBMA hydrogel, (b) gelation process of GO-PSBMA nanocomposite hydrogel. 